06,13

\title{
Определение стационарного тока утечки в структурах с пленками сегнетоэлектрической керамики
}

\author{
() Ю.В. Подгорный, К.А. Воротилов, А.С. Сигов \\ Московский технологический университет (МИРЭА), \\ Москва, Россия \\ E-mail: podgsom_2004@mail.ru
}

\begin{abstract}
Исследованы стационарные токи утечки конденсаторных структур с сегнетоэлектрическими золь-гель пленками цирконата-титаната свинца (PZT), сформированными на кремниевых подложках с нижним Pt электродом. Установлено, что независимо от толщины пленки PZT структуры Pt/PZT/Hg характеризуются наличием выпрямляющего контакта, подобного $p$-n-переходу. Стационарный ток утечки в проводящем направлении увеличивается с уменьшением толщины пленки и определяется проводимостью объема сегнетоэлектрика.
\end{abstract}

Работа выполнена при финансовой поддержке Минобрнауки России в рамках базовой части государственного задания в сфере научной деятельности, проект № 3.5726.2017/БЧ.

DOI: 10.21883/FTT.2018.03.45539.02D

\section{1. Введение}

Интеграция сегнетоэлектрических тонких пленок с полупроводниковыми технологиями позволяет создать новые классы устройств приема, обработки и хранения информации, например $[1,2]$. Корректное определение стационарного тока утечки необходимо как для объективного контроля качества сегнетоэлектрических структур, так и для идентификации механизмов переноса носителей заряда. Обычно используют два метода измерения зависимостей ток-напряжение: динамический (voltage-ramp) и статический (voltage-step) [3]. Результаты определения тока утечки динамическим методом зависят от скорости развертки напряжения, кроме того не исключена существенная погрешность, обусловленная током восстановления поляризации, несмотря на предварительную поляризацию пленки [4,5].

Суть статического метода состоит в наблюдении тока релаксации (заряда) при заданном уровне постоянного напряжения до полной его стабилизации, значение которого обычно и принимают за стационарный ток утечки. Зачастую данное состояние оказывается недостижимым из-за деградации пленки при длительном воздействии постоянного напряжения - ток, не достигнув стационарного состояния, начинает расти. Минимизировать недостатки статического метода, то есть сократить время наблюдения и повысить точность измерения, позволяет использование адекватной модели тока релаксации [6]. Однако вопросы корректного описания зависимостей ток-электрическое поле для различных полярностей напряжения смещения остаются невыясненными.

Целью настоящей работы был анализ зависимостей стационарного тока утечки от напряжения и поиск адекватных моделей, описывающих данные зависимости в типичных структурах, используемых в полупроводниковой технологии: $\mathrm{Si}-\mathrm{SiO}_{2}-\mathrm{TiO}_{2}-\mathrm{Pt}-\mathrm{PZT}$ с использованием стандартной методики контроля с $\mathrm{Hg}$ зондом.

\section{2. Методика эксперимента}

Пленки $\mathrm{Pb}\left(\mathrm{Zr}_{0.48} \mathrm{Ti}_{0.52}\right) \mathrm{O}_{3}$ формировали на подложках $\mathrm{Si}-\mathrm{SiO}_{2}-\mathrm{TiO}_{2}-\mathrm{Pt}$ методом послойного химического осаждения из растворов в соответствии с методикой [7]. Электрические измерения проводили с помощью зонда (MDC 802-150) с диаметром ртутного контакта $760 \mu \mathrm{m}$. Измерение характеристик петель сегнетоэлектрического гистерезиса осуществляли на установке ТF Analyzer $2000 \mathrm{E}$ на частоте $100 \mathrm{~Hz}$ при амплитуде напряженности электрического поля $E_{m} \sim 180-235 \mathrm{kV} / \mathrm{cm}$. Зависимости тока утечки $J(E, t)$ от напряженности электрического поля $E$ и времени $t$ измеряли пикоамперметром Hewlett Packard (Agilent) 4140B.

\section{3. Результаты}

В таблице приведены основные параметры петель гистерезиса структур с пленками PZT: $d-$ толщина пленки; $E_{c r}-$ коэрцитивное поле; $P_{r}^{+}$и $P_{r}^{-}-$положительная и отрицательная динамическая остаточная поляризация; $P_{r \text { rel }}^{+(-)}-$значения заряда остаточной поляризации после релаксации за $1 \mathrm{~s} ; P_{t}$ - поляризация на вершинах петли гистерезиса.

На рис. 1 и 2 показаны экспериментальные значения (символы) и моделирующие зависимости $J(t)$ тока релаксации (заряда) металл-сегнетоэлектрик-металл (MCM) структуры с пленкой PZT толщиной $1067 \mathrm{~nm}$ при различных значениях напряженности электрического поля отрицательной и положительной полярности (на верхнем $\mathrm{Hg}$ электроде) соответственно. Аналогичный вид имеют зависимости $J(t)$ для МСМ-структур с пленками другой толщины. Моделирование во временной области зависимостей $J(t)$ суммой двух и/или трех экспоненциальных (низкочастотных) функций [6] позволяет с приемлемой точностью определять стационарный ток 
Характеристики сегнетоэлектрического гистерезиса

\begin{tabular}{c|c|c|c|c|c|c|c|c}
\hline$d$ & $E_{m}$ & $E_{c r}^{+}$ & $E_{c r}^{-}$ & $P_{t}$ & $P_{r}^{+}$ & $P_{r}^{-}$ & $P_{r \text { rel }}^{+}$ & $P_{\text {rrel }}^{-}$ \\
\hline $\mathrm{nm}$ & \multicolumn{5}{|c}{$\mathrm{kV} / \mathrm{cm}$} & \multicolumn{5}{c}{$\mu \mathrm{C} / \mathrm{cm}^{2}$} \\
\hline 213 & 235 & 73 & -69 & 40 & 25.3 & -27.1 & 18.4 & -13.4 \\
\hline \multirow{2}{*}{552} & 181 & 48 & -52 & 38 & 24.0 & -23.8 & 21.1 & -20.7 \\
& 226 & 50 & -53 & 40 & 25.1 & -24.7 & 21.9 & -21.4 \\
\hline \multirow{2}{*}{1067} & 187 & 51 & -43 & 40 & 25.0 & -26.4 & 21.1 & -23.8 \\
& 234 & 53 & -45 & 43 & 26.1 & -27.2 & 22.0 & -24.3 \\
\hline \multirow{2}{*}{1165} & 172 & 41 & -43 & 35 & 22.1 & -20.7 & 18.9 & -15.1 \\
& 214 & 44 & -45 & 37 & 23.4 & -21.8 & 20.1 & -16.2
\end{tabular}

утечки при ограниченной продолжительности наблюдений

$$
J(t)=\sum_{i=1}^{k} J_{m i} \cdot \exp \left(-t / \tau_{i}\right)+J_{s s},
$$

где $\tau_{i}-$ время релаксации; $J_{m i}-$ амплитуда $i$-ой экспоненциальной составляющей тока релаксации; $J_{s s}-$ стационарный ток; $k$ - число экспоненциальных функций (2 или 3) определяется началом по времени $t_{s t}$ диапазона моделирования. С целью повышения точности за счет уменьшения случайной погрешности измерений (особенно в начале диапазона наблюдений) стартовые значения $t_{s t}$ использовались с дискретностью $\sim 5 \mathrm{~s}$ в пределах от 11 до $28 \mathrm{~s}$ и от 33 до $60 \mathrm{~s}$ при моделировании суммой трех и двух экспоненциальных функций соответственно. Средние для всех образцов значения времени релаксации составили 360, 60 и $11 \mathrm{~s}$ при отрицательной полярности, а при положительной полярности 264 и $54 \mathrm{~s}$.

Особенность полученных зависимостей состоит в том, что стационарный ток $J_{s s}$ с ростом напряжения отрицательной полярности увеличивается (см. рис. 1), в то время как с увеличением положительного смещения $(\geq 50 \mathrm{kV} / \mathrm{cm})$ ток $J_{s s}$ остается практически неизменным (см. вставку на рис. 2).

Измерения каждой следующей $J(t)$ зависимости при положительной полярности выполняли на новой структуре $\mathrm{Pt} / \mathrm{PZT} / \mathrm{Hg}$ для исключения эффекта памяти, который может оказать влияние в случае определения зависимостей $J(t, E)$ на одной структуре при постепенном ступенчатом повышении напряжения. На рис. 2 для пленки $1067 \mathrm{~nm}$ показаны четыре зависимости тока релаксации для трех значений поля положительной полярности, иллюстрирующие отсутствие закономерности в зависимости стационарного тока от действующего поля. Кривые $J(t)$ для полей 70 и $140 \mathrm{kV} / \mathrm{cm}$ расположены между кривыми $J(t)$, полученными при напряженности $234 \mathrm{kV} / \mathrm{cm}$ на двух различных структурах, что связано с дисперсией измерений и свойств образцов.

Таким образом, вид полученных зависимостей аналогичен вольт-амперной характеристике (BAX) полупроводникового диода с $p-n$-переходом, включенного в проводящем или запирающем направлении, что и определило выбор моделирующей функции

$$
E(J)=\eta \Phi_{T} \cdot \ln \left(\frac{J}{J_{0}}+1\right)+k_{b} \cdot J_{s s}^{n} .
$$

При $n=1$ уравнение (2) представляет ВАХ полупроводникового диода с $p-n$-переходом, включенным в

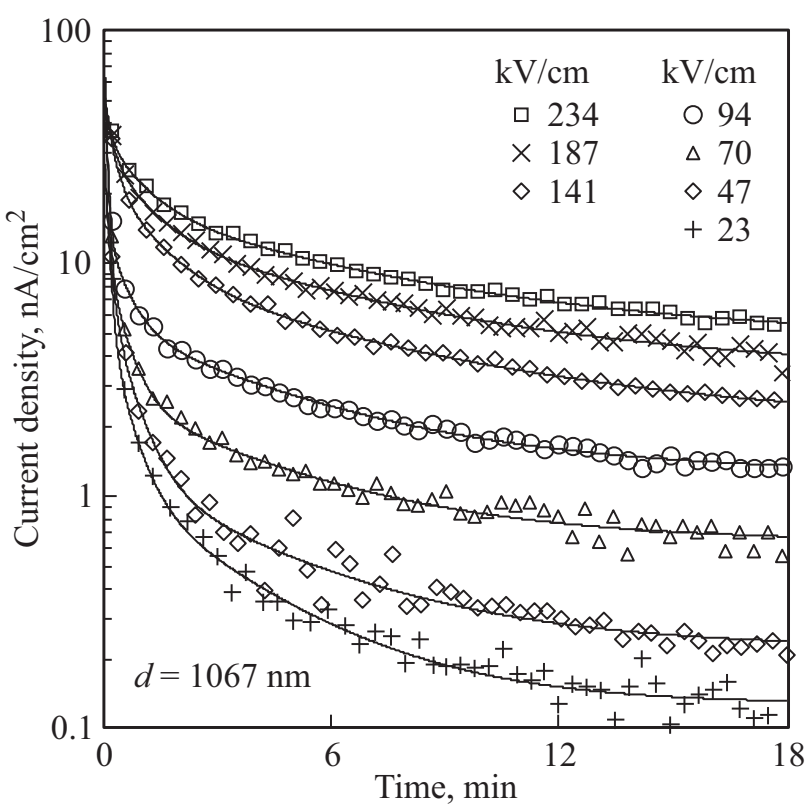

Рис. 1. Зависимости тока релаксации от времени $J(t)$ при постоянном напряжении отрицательной полярности (в проводящем направлении) для МСМ-структуры с пленкой PZT $1067 \mathrm{~nm}$

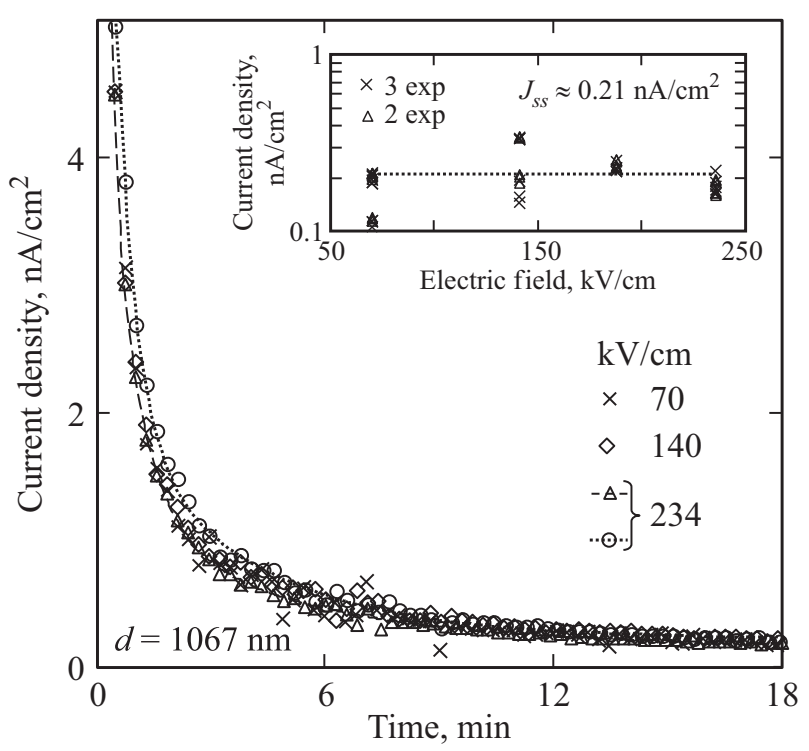

Рис. 2. Зависимости тока релаксации от времени $J(t)$ при постоянном напряжении положительной полярности (в запирающем направлении) для МСМ-структуры с пленкой PZT $1067 \mathrm{~nm}$. На вставке показаны значения стационарного тока при электрическом поле $\geq 50 \mathrm{kV} / \mathrm{cm}$. 


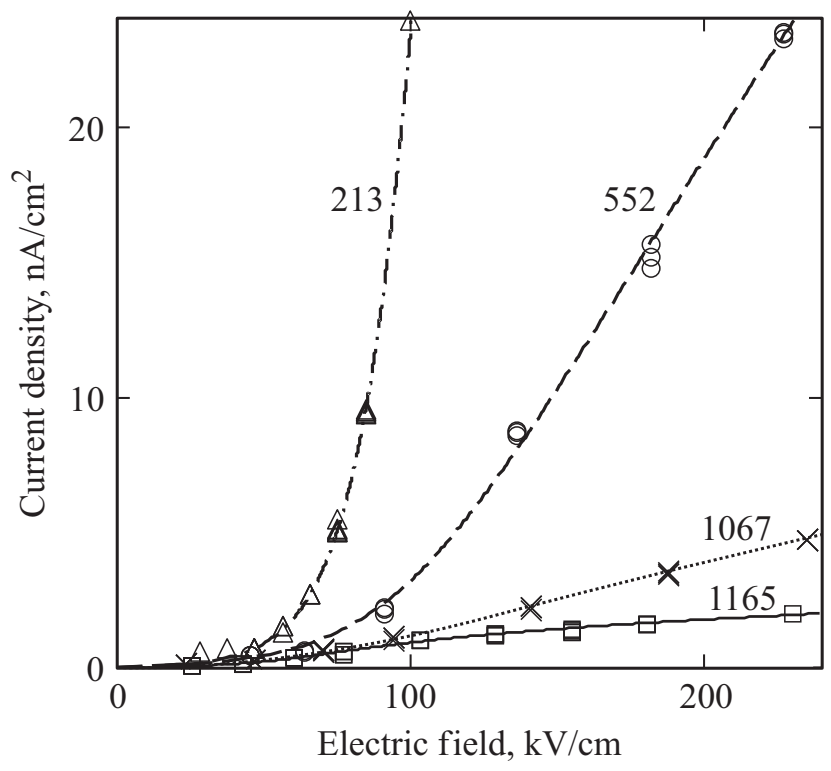

Рис. 3. Характеристики стационарного тока (экспериментальные и моделируемые с помощью уравнения (2) в проводящем направлении) для МСМ-структур с пленками PZT толщиной $213,512,1067$ и $1165 \mathrm{~nm}$.

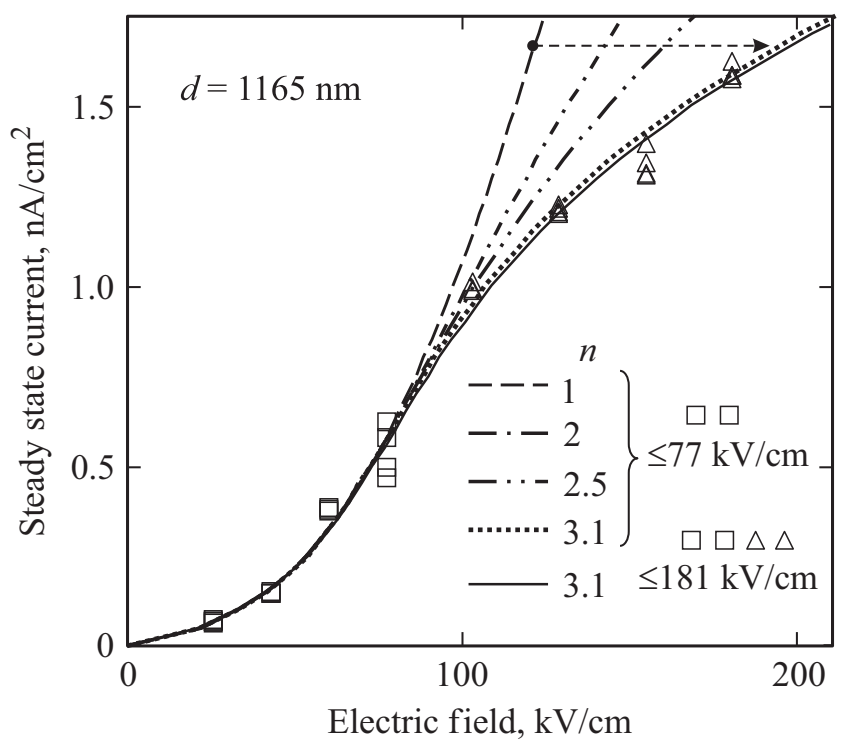

Рис. 4. Влияние коэффициента $n$ на моделирование ВАХ в проводящем направлении для МСМ-структуры с пленкой толщиной $1165 \mathrm{~nm}$.

проводящем направлении, в котором $\Phi_{T}$ - температурный потенциал $\mathrm{kV} / \mathrm{cm} ; \eta-$ фактор неидеальности $(\sim 2-3)$ [8]; $J_{0}$ - тепловой ток; $k_{b}-$ калибровочный коэффициент. Показатель степени $n$ учитывает нелинейную зависимость от действующего поля проводимости в объеме сегнетоэлектрической пленки с низкой концентрацией носителей, что обусловлено совокупностью различных действующих механизмов переноса $[4,9]$. При $n=1$ (линейная база) имеем $k_{b}=r_{b}$ - омическое со- противление объема сегнетоэлектрической пленки („базы“). Определение параметров модели с использованием экспериментальных данных выполнялось как в $I-U$, так и в $J-E$ координатах в различных диапазонах действующего поля.

На рис. 3 показаны экспериментальные результаты и соответствующие моделирующие $J_{s s}(E)$ зависимости стационарного тока при отрицательной полярности. Моделирование в различных диапазонах электрического поля показывает, что сопротивление объема пленки при напряженности поля ниже $\sim 70-80 \mathrm{kV} / \mathrm{cm}$ имеет омический характер $(n=1)$, а нелинейность проявляется при более высоких полях. Рис. 4 на примере пленки с $d=1165 \mathrm{~nm}$ иллюстрирует эффективность параметра $n$ в модели стационарного тока. По экспериментальным данным в диапазоне поля $\leq 77 \mathrm{kV} / \mathrm{cm}$ методом нелинейной регрессии определялись только параметры $\eta \Phi_{T}, J_{0}$ и $k_{b}$, при этом коэффициент нелинейности $n$ искусственно изменяли от 1 до 3.1. Соответствующая кривая аппроксимации при $n=3.1$ практически полностью совпадает с аппроксимирующей кривой, полученной при моделировании во всем диапазоне данных (до $\sim 180 \mathrm{kV} / \mathrm{cm})$ и определении всех параметров методом нелинейной регрессии.

Значения плотности теплового тока $J_{0}$ и температурного потенциала $\eta \Phi_{T}\left(3 \eta \Phi_{T}-\right.$ поле, при котором происходит отпирание $p-n$-перехода), практически не зависят от толщины пленки в пределах погрешности измерения, т.е. определяют электрические характеристики интерфейсных слоев МСМ-структур. Введение в моделирующую функцию степенной зависимости падения напряжения на объеме пленки („базе“) от протекающего тока позволяет получить идентичные значения моделирующих параметров во всем диапазоне действующего поля.

\section{4. Заключение}

Экспериментальные исследования стационарного тока утечки при внешнем поле различной полярности показали, что структуры $\mathrm{Pt} / \mathrm{PZT} / \mathrm{Hg}$ обладают выпрямляющим контактом. Исходя из того, что объем пленки PZT имеет проводимость $p$-типа $[1,3]$, можно полагать, что на границе с $\mathrm{Hg}$ электродом существует область с проводимостью $n$-типа. Значения плотности теплового тока $J_{0}$ и температурного потенциала $\eta \Phi_{T}$ в пределах погрешности измерений не зависят от толщины пленки, свидетельствуя о том, что они в действительности определяют электрические характеристики интерфейсных слоев МСМ-структур. В то же время, стационарный ток имеет сильную зависимость от толщины пленки, что предполагает увеличение концентрации носителей в объеме пленки PZT с уменьшением её толщины. 


\section{Список литературы}

[1] J.F. Scott. Science 315, 954 (2007).

[2] K.A. Vorotilov, A.S. Sigov. Physics of the Solid State 54, 5, 894 (2012).

[3] G.W. Dietz, M. Schumacher, R. Waser, S.K. Streiffer, C. Basceri, A.I. Kingon. J. Appl. Phys. 82, 5, 2359 (1997).

[4] A. Sigov, Yu. Podgorny, K. Vorotilov, A. Vishnevskiy. Phase Trans. 86, 1141 (2013).

[5] Yu. Podgorny, K. Vorotilov, A. Sigov. J. Appl. Phys. Lett. 105, 182904 (2014).

[6] Yu. Podgorny, K. Vorotilov, A. Sigov. AIP Advances 6, 095025 (2016).

[7] N.M. Kotova, K.A. Vorotilov, D.S. Seregin, A.S. Sigov. Inorganic Materials. 50, 6, 612 (2014).

[8] S.M. Sze, K.Ng Kwok. Physics of Semiconductor Devices, John Wiley \& Sons, Hoboken, New Jersey (2007). 119 p.

[9] M. Dawber, K.M. Rabe, J.F. Scott. Rev. Mod. Phys. 77, 1083 (2005). 